\title{
JUVENTUD RURAL EN CHILE ¿PROBLEMA O SOLUCIÓN?
}

DANIEL DUHART*

LA JUVENTUD RURAL REPRESENTA un sector clave de la población juvenil chilena y la forma de cómo se aborda a este grupo etario puede ser determinante para su accionar en el futuro, así como para el desarrollo de las zonas rurales en general. De acuerdo con las nuevas teorías de desarrollo rural, el equilibrio urbano-rural asoma como un elemento esencial para el logro de una calidad de vida adecuada, tanto en el campo como en la ciudad. Cada vez más las ciudades dependen del campo, y de cómo éstas manejan y conservan los recursos, la cultura y los espacios naturales. Además, el mismo concepto de una «ruralidad» pequeña y aislada v/s una urbanidad extrema y «gigante» está siendo cuestionado, y nuevas propuestas y definiciones sobre lo rural centrado en el territorio regional están siendo adoptadas (Echeverri y Ribero, 2002; Arbab, 1991). Una juventud rural con conciencia ciudadana aparece como un actor social importante en este contexto de cambios en su entorno.

Sin embargo, un grave problema de enfoque amenaza a muchos programas y visiones que abordan a la juventud rural. Generalmente la visión que existe es que la juventud rural representa un problema que debe solucionarse, un costo inevitable del llamado proceso de modernización, expresado en carencias económicas, sociales y educaciona-

* Licenciado en Historia y Magíster en Estudios Sociales y Políticos Latinoamericanos. Actualmente docente de la Universidad Alberto Hurtado y consultor del CIDE. E-Mail: dduhart@cide.cl. 
les. Aunque un diagnóstico así puede no estar lejos de los desafíos que deben enfrentar, impide visualizar los potenciales que éstos puedan tener. En este sentido, visualizar a la juventud rural como una solución y un aporte al desarrollo local, regional y nacional, a través de sus iniciativas individuales y colectivas, puede ser el catalizador de interesantes procesos, beneficiándose la sociedad en su búsqueda de un desarrollo más equitativo y sustentable.

\section{I. ¿POR QUÉ OCUPARSE DE LA JUVENTUD RURAL?}

Como reflexión inicial es interesante citar esta pregunta que la División de Desarrollo Social de la CEPAL realizó al comienzo del seminario internacional «Juventud rural, modernidad y democracia en América Latina» en marzo de 1996 (CEPAL, 1996:18-22). Esta pregunta es esencial, ya que representa la base desde la cual se justifica la reflexión y acción hacia ellos. Sin embargo, el tono de la misma pregunta ya refleja el modelo mental que la juventud es un problema, un escándalo social (como se plantea en las teorías de política social), del cual debemos preocuparnos. Como dijimos, planteamos que el enfoque debe ser distinto, positivo, y justamente por eso debemos ocuparnos de la juventud rural, para ver sus potenciales, en especial frente a la invisibilidad que sufren a los ojos de la sociedad en general.

Ante esta pregunta, uno de los principales argumentos hoy en día, desde una perspectiva más ligada al desarrollo económico y las políticas sociales, afirma que es importante adoptar un enfoque etario en las políticas sociales para de este modo estructurar políticas de desarrollo rural integrales y centradas en las necesidades reales de las personas. Desde esta perspectiva, ocuparse de la juventud rural representaría una evolución en el diseño de políticas, pudiendo afinar con más efectividad, eficiencia y eficacia su accionar. Existiría un consenso que se están produciendo una serie de transformaciones en las economías de la región latinoamericana que podrían influir mucho en la agricultura y la sociedad rural, dejando a la juventud rural vulnerable al contar con un apoyo estatal aún deficiente, por lo que más y mejores programas enfocados hacia ellos serían vitales (CEPAL, 1996:21). Ocuparse de la juventud rural sería una forma de reducir la brecha de la equidad, citando nuevamente el lenguaje de la CEPAL.

Estas afirmaciones y conclusiones son sin duda ciertas, y muy importantes, debiendo ser consideradas en el análisis de la realidad de los jóvenes rurales. Sin embargo, pueden ser limitantes si tan solo nos 
concentramos en ellas y no vemos otras dimensiones ligadas a la juventud rural más allá de políticas de «parche» ante las crisis económicas post-ajuste estructural. Debemos analizar los potenciales y oportunidades que ofrece la juventud rural.

En primer lugar, existiría hoy en día una coyuntura histórica clave para redefinir la orientación del desarrollo rural y social en general, cuando la región latinoamericana aún posee una importante población juvenil, frente al proceso de transición demográfica y envejecimiento de la sociedad. Es posible que en cincuenta años más la situación sea diferente, como indica la tendencia (Dirven, 2002).

En segundo lugar, a consecuencia de los procesos gemelos de globalización y localización, la generación juvenil actual posee una familiaridad con la cultura y valores de la modernidad mucho mayor que la generación de sus padres (así como una mayor educación formal), y a la vez mantiene un arraigo con la cultura local: es un potencial actor social híbrido, clave para el futuro desarrollo de las comunas, regiones y países latinoamericanos, pudiendo moverse entre distintas culturas y en este sentido, ofreciendo características interesantes para la búsqueda de un modelo de desarrollo más armónico con el medio ambiente y las identidades culturales locales.

Y esto no lleva a nuestra idea central. De acuerdo con lo que planteamos al inicio, la juventud rural tiene un rol vital no sólo en lo que concierne a sus propios desafíos y expectativas, sino a los de la sociedad en general, especialmente frente la relación entro lo rural y lo urbano. Es urgente hoy en día una redefinición de esta relación, no sólo para mejorar la calidad de vida en las ciudades, sino para reorientar el paradigma de desarrollo general. En la actualidad, el concepto de desarrollo regional, centrado en el territorio y la identidad regionales/locales, se ha consolidado como un concepto líder para las futuras políticas y programas de desarrollo, y en esta coyuntura los jóvenes rurales pueden entregar y ayudar mucho, siendo actores principales del proceso, como veremos en las siguientes páginas. Pero antes debemos analizar los potenciales y desafíos que presenta la juventud rural particularmente en Chile, cuál es nuestro ámbito de reflexión.

\section{Potenciales y desafíos de la JUVEntUd RURAL EN ChILE}

Existe bastante información que describe la realidad demográfica, educacional, social, económica, cultural, etc., de la juventud rural en Chile, pero en esta sección deseamos destacar los aspectos que refle- 
jan el gran potencial que poseen, y los desafíos que surgen a consecuencia. Pero para empezar, debemos contemplar el universo de población sobre el cual estamos reflexionando.

Tabla 1

Población juvenil 2002

\begin{tabular}{|l|c|c|c|}
\hline GRUPOS DE EDAD & $\begin{array}{c}\text { TOTAL } \\
\text { AMBOS SEXOS }\end{array}$ & $\begin{array}{c}\text { URBANA } \\
\text { AMBOS SEXOS }\end{array}$ & $\begin{array}{c}\text { RURAL } \\
\text { AMBOS SEXOS }\end{array}$ \\
\hline Total País & 15.116 .435 & 13.090 .13 & 2.026 .322 \\
\hline 10 a 14 años & 1.422 .452 & 1.225 .513 & 196.939 \\
\hline 15 a 29 años & 3.674 .239 & 3.230 .653 & 443.586 \\
\hline
\end{tabular}

Fuente: INE, 2003.

Podemos observar en esta tabla que la población juvenil (15-29 años) total del país es un $24,3 \%$ del total de la población, con un $88 \%$ urbano y un $12 \%$ rural. A su vez, la juventud rural representa un 3,0\% del total de la población del país. No deja de ser un porcentaje considerable si recordamos que muchos jóvenes rurales han emigrado a la ciudad, engrosando la población juvenil urbana, pero cultural y socialmente representando a la ruralidad (o una identidad híbrida). Además, el concepto de ruralidad visto desde una perspectiva cultural amplía aún más este universo, abarcando a ciudades medianas, pero que cultural, social, económica y ambientalmente están totalmente integradas con el territorio rural. De todos modos, si ya un $12 \%$ de la población juvenil es considerada clásicamente rural, esto ya cuestiona la imagen tradicionalmente urbana de la juventud chilena, dejando claro que debe existir una política especial para ese $12 \%$ de la población juvenil.

Un potencial interesante en la juventud rural se presenta en las cifras de educación, superando con creces a la generación de sus padres. La siguiente tabla refleja la evolución en el promedio de años de estudio que ha tenido la juventud rural chilena en los últimos quince años aproximadamente.

Podemos observar en la tabla que ha habido un aumento en 1.5 años promedio para ambos sexos en el ámbito rural entre 1987 y el año 2000, destacando las mujeres, que han llegado a 9.2 años de estudio promedio, en contraste con los 8.7 de los hombres. De todos modos, aún se presenta un promedio menor en relación con la juventud urbana, que tiene 10.6 años, reflejando la mayor presencia de la educación media y de doble jornada en las ciudades. En este sentido, como decíamos más arriba, un desafío para la política social sería encon- 
trar una forma de hacer llegar la educación media al campo, o un sistema de educación a distancia que pueda suplir esta necesidad y a su vez adecuarse al ciclo de vida rural (trabajo en el predio, trabajo temporal, etc.).

Tabla 2

Promedio de años de estudio de la población de 15 a 24 años según sexo, zonas urbanas y rurales, Chile 1987-2000

(en promedios)

\begin{tabular}{|l|c|c|c|c|c|c|}
\hline AÑO & \multicolumn{3}{|c|}{ ZONAS URBANAS } & \multicolumn{3}{c|}{ ZONAS RURALES } \\
\hline & \multicolumn{2}{|c|}{$\begin{array}{c}\text { PROMEDIO DE AÑOS DE } \\
\text { INSTRUCCIÓN }\end{array}$} & \multicolumn{3}{c|}{$\begin{array}{c}\text { PROMEDIO DE AÑOS DE } \\
\text { INSTRUCCIÓN }\end{array}$} \\
\hline & $\begin{array}{c}\text { AMBOS } \\
\text { SEXOS }\end{array}$ & HOMBRES & MUJERES & $\begin{array}{c}\text { AMBOS } \\
\text { SEXOS }\end{array}$ & HOMBRES & MUJERES \\
\hline 1987 & 9.9 & 9.9 & 10.0 & 7.4 & 7.1 & 7.6 \\
\hline 1990 & 10.1 & 10.0 & 10.2 & 7.9 & 7.6 & 8.1 \\
\hline 1994 & 10.4 & 10.4 & 10.5 & 8.2 & 8.0 & 8.4 \\
\hline 2000 & 10.6 & 10.6 & 10.7 & 8.9 & 8.7 & 9.2 \\
\hline
\end{tabular}

Fuente: CEPAL, 2002.

Donde se observa el mayor contraste en cuanto a educación formal entre la generación actual de jóvenes rurales y sus padres y abuelos, es en la condición de alfabetismo, como se refleja en la tabla 3. A pesar que aún existe un muy pequeño contraste entre la juventud rural urbana y rural, con un $99 \%$ y un $97 \%$ de porcentaje de alfabetismo respectivamente, es incluso mayor la diferencia entre los jóvenes rurales y sus padres y abuelos, bajando el porcentaje de alfabetismo a medida que aumenta la edad de la población rural (llegando a entre un $75 \%$ y un 55\% para la población de adultos mayores).

En este sentido, ha habido un gran avance en cuanto a educación formal entre la juventud rural, el cual representa un tremendo potencial de desarrollo como capital humano. El desafío, sin embargo, es analizar la calidad de aquella educación, ya que además de leer y escribir debe comprenderse la lectura. Se debe verificar también la pertinencia del actual currículo de educación en el mundo rural, ya que existe una sensación general que ésta no responde a los intereses de los jóvenes rurales y sus proyectos de vida ligados al campo. La educación formal estaría basada en un paradigma de juventud urbana, y sus intereses y proyectos de vida. 
Tabla 3

Población de 10 años o más, condición de alfabetismo, porcentaje y sexo, según área urbana y rural y grupos de edad, Chile

\begin{tabular}{|l|c|c|c|}
\hline \multirow{2}{*}{ TOTAL PAÍS } & \multicolumn{3}{|c|}{ PORCENTAJE DE ALFABETISMO } \\
\cline { 2 - 4 } & AMBOS SEXOS & HOMBRES & MUJERES \\
\hline Urbana y rural & $\mathbf{9 5 , 7 9}$ & $\mathbf{9 5 , 8 3}$ & $\mathbf{9 5 , 7 5}$ \\
\hline Urbana & $\mathbf{9 6 , 8 1}$ & $\mathbf{9 6 , 9 9}$ & $\mathbf{9 6 , 6 5}$ \\
\hline 10 a 14 años & 96,52 & 96,28 & 96,77 \\
\hline 15 a 24 años & 99,15 & 99,00 & 99,30 \\
\hline 25 a 34 años & 97.21 & 98,53 & 98,93 \\
\hline Rural & $\mathbf{8 9 , 1 6}$ & $\mathbf{8 9 , 1 1}$ & $\mathbf{8 9 , 2 2}$ \\
\hline 10 a 14 años & 95,37 & 94,81 & 95,99 \\
\hline 15 a 24 años & 97,80 & 97,44 & 98,23 \\
\hline 25 a 34 años & 96,1 & 95,48 & 96,78 \\
\hline 35 a 44 años & 92,60 & 92,15 & 93,14 \\
\hline 45 a 54 años & 87,56 & 87,9 & 87,13 \\
\hline 55 a 64 años & 76,30 & 76,92 & 75,56 \\
\hline 65 a 74 años & 68,34 & 69,29 & 67,22 \\
\hline 75 a 84 años & 60,65 & 62,44 & 58,77 \\
\hline 85 a 94 años & 58,70 & 60,15 & 57,53 \\
\hline 95 a 104 años & 57,66 & 68,58 & 50,94 \\
\hline
\end{tabular}

Fuente: INE, 2003.

La siguiente serie de conclusiones obtenidas de dos entrevistas focales (focus group), ${ }^{1}$ realizadas por Sonia Zapata con jóvenes rurales trabajadores y dirigentes de la VII Región, confirma estas apreciaciones: i) Los jóvenes perciben el proceso educativo del área rural con un corte urbano y, en el fondo, el propósito concreto es orientar al joven a la educación superior. ii) No contribuye a que el joven pueda desenvolverse o desarrollarse mejor en el campo. iii) Existe una fuerte desconfianza y baja autoestima, reflejado en la idea que ellos poseen falencias educacionales en comparación con los jóvenes urbanos, desertando muchos de ellos por sentirse incapaces de aprender. iv) También se sienten seducidos por los medios de comunicación, que les presentan una ciudad atractiva donde quieren llegar lo antes posible. v) Muchos emigran a la ciudad y engrosan las filas de desocupados y marginados, ya que su nivel de educación no les permite obtener un trabajo. vi) Estos jóvenes caen bajo el efecto de deslumbramiento, en

1 Uno, con sujetos tipos adolescentes trabajadores rurales e hijos de pequeños propietarios, y el otro, con sujetos tipos dirigentes rurales. 
que pierden su identidad campesina y permanecen en la ciudad, con sus expectativas revolucionadas ante una soñada, pero irreal mejor vida (Zapata, 2000:98-99).

Además, no sólo esta educación sería inadecuada para los jóvenes rurales y sus proyectos de vida, sino que también existiría una falta de fuentes de estudio y opciones, debido al aislamiento y lejanía en que viven muchos con respeto a las ciudades, así como al ciclo de vida rural, por el cual deben trabajar en el predio de sus padres. Un estudio con jóvenes rurales de la VI, VII y VIII regiones confirma esta realidad.

Debería haber más fuentes de estudio para poder estudiar, así hay gente como ellos mismos no pueden salir lejos entonces podrían haber por acá centros de estudios... les queda lejos y no hay cómo venirse (INJUV, 1998:80).

Yo estaba estudiando, llegué hasta tercero y no más, estaba estudiando electrónica, pero tuve problemas en la casa y no pude seguir estudiando, así que tuve que retirarme (INJUV, 1998:78).

...entré muy lolo al trabajo, compartía tres días al trabajo y tres días al colegio... así que a duras penas llegué a séptimo... cuando llegué a entrar a octavo dije que no porque ya seguí ganando las monedas, así que ahora ya no creo que vuelva a seguir... (INJUV, 1998:78).

Este proceso de deserción escolar lo podemos observar en la siguiente tabla, donde se aprecia que aún la deserción es bastante mayor en las zonas rurales que en las urbanas.

Tabla 4

Tasa de deserción escolar entre los jóvenes de 15 a 19 años, según sexo, zonas urbanas y rurales, 1990-2000 (en porcentajes)

\begin{tabular}{|c|c|c|c|c|c|c|c|c|c|}
\hline Año & \multicolumn{3}{|c|}{ Nacional } & \multicolumn{3}{c|}{ Zonas urbanas } & \multicolumn{3}{c|}{ Zonas rurales } \\
\cline { 2 - 10 } & Total & Hombres & Mujeres & Total & Hombres & Mujeres & Total & Hombres & Mujeres \\
\hline 1990 & 27 & 27 & 28 & 21 & 20 & 21 & 56 & 57 & 56 \\
\hline 2000 & 17 & 17 & 17 & 14 & 14 & 14 & 32 & 33 & 30 \\
\hline
\end{tabular}

Fuente: CEPAL, 2002.

A pesar que la tasa de deserción ha disminuido en los últimos diez años, sigue siendo el doble que en las zonas urbanas, con un 32\% para ambos sexos, v/s un 17\% en el sector urbano. Destaca también que en 
el ámbito rural son más los hombres que desertan (un 33\% v/s un $30 \%$, respectivamente), reflejando la mayor tendencia de las mujeres a completar sus estudios, mientras que muchos hombres deben desertar por razones laborales, ya sea para trabajar en el predio familiar, o en trabajo salariado temporal o permanente (como vimos en las opiniones citadas más arriba). En este sentido, más que buscar formas de extender el sistema de educación media y la jornada escolar completa a las zonas rurales, intentando urbanizar su estilo de vida, podría buscarse un sistema educacional que se adapte a las necesidades y condiciones del ciclo de vida rural (Durston, 1998), basándose en el potencial que estos jóvenes poseen para el futuro desarrollo de sus vidas, sus comunidades y sus regiones.

Otra dimensión importante de los jóvenes rurales tiene que ver con el trabajo, elemento central de su ciclo de vida, el cual en muchas ocasiones está ligado a la migración, como es el caso de los temporeros. La siguiente tabla refleja las ventajas y desventajas de este tipo de trabajo de acuerdo con opiniones de jóvenes rurales de la VI, VII y VIII regiones.

Tabla 5

Juventud rural y trabajo temporal

\begin{tabular}{|l|l|}
\hline \multicolumn{1}{|c|}{ VENTAJAS } & \multicolumn{1}{|c|}{ DESVENTAJAS } \\
\hline Entrega ingresos inmediatos. & Es poco trabajo, dura poco. \\
\hline $\begin{array}{l}\text { Es una oportunidad para permanecer } \\
\text { en el campo y no tener que emigrar } \\
\text { a la ciudad. }\end{array}$ & Es mal pagado y difícil de encontrar. \\
\hline $\begin{array}{l}\text { Se aprenden cosas que sirven } \\
\text { para encontrar más trabajo. }\end{array}$ & Es sacrificado. \\
\hline $\begin{array}{l}\text { Entrega experiencia e ideas para nuevos } \\
\text { trabajos. }\end{array}$ & Es inseguro, no tiene contrato. \\
\hline Independencia, libertad. & $\begin{array}{l}\text { No pueden quejarse y organizarse. } \\
\text { No conocen sus derechos y temen } \\
\text { a los patrones. }\end{array}$ \\
\hline Hay trabajo para las mujeres. & Los pesticidas dañan la salud. \\
\hline
\end{tabular}

Fuente: basado en INJUV, 1998.

Podemos ver en esta tabla que el trabajo temporal es ambiguo, pues ofrece tanto ventajas como desventajas para los jóvenes rurales. Por un lado entrega independencia y movilidad social, así como un ingreso rápido y una sensación de libertad. A su vez ofrece oportunidades 
para las mujeres, aprendizaje y experiencia, así como es una alternativa laboral para permanecer en el campo sin tener que emigrar definitivamente. Sin embargo, por otro lado es un trabajo mal pagado y duro, que genera inseguridad y dependencia, no siendo una alternativa de proyecto de vida que fortalezca las capacidades y autoestima de los jóvenes rurales. Es una opción inmediatista, como muchas opciones en el modelo de sociedad de mercado actual, pero que no permite a los jóvenes convertirse en actores sociales reales en su entorno, pues dificulta su organización y acción colectiva.

En un estudio cualitativo con jóvenes temporeros, De la Maza llega a la siguiente tipología de juventud temporera, reflejando que muchos jóvenes se insertan en este tipo de trabajo desde muy temprana edad, acompañando a sus padres en las jornadas laborales: a) Jóvenes quienes ingresaron a trabajar entre los 7 y 9 años, asociado a faenas que se realiza por parte de toda la familia. b) Jóvenes que ingresaron a trabajar en la temporada entre los 12 y 14 años, distinguiéndose dos trayectorias: i) La de «trabajadores estacionales», aquellos que aprovechan las vacaciones de verano como una forma de aumentar los ingresos familiares. ii) Los «trabajadores temporeros tempranos», aquellos que terminaron octavo básico y carecen de oportunidades tanto de continuidad escolar como de alternativas laborales distintas al trabajo temporero (De la Maza, 1999).

Por este motivo, es una realidad que debe enfrentarse desde la niñez y pre-juventud, ofreciendo opciones diferentes, pues no permite a los jóvenes completar su educación y desarrollarse en otras esferas. Analizándolo desde otra perspectiva, y tomando en cuenta las ventajas que ofrece el trabajo temporal, implica adecuar el sistema educacional a este estilo de vida rural, como ya mencionamos antes, con un sistema educacional que se adapte al ciclo de vida rural (trabajo familiar, relaciones intergeneracionales, etc.).

Otra dimensión muy importante para una política social dirigida a la juventud rural es la participación social, en especial si se desea orientar ésta hacia el fortalecimiento de actores sociales. En este contexto, podemos identificar interesantes características de la participación juvenil rural, las cuales nos reflejan algunas de las capacidades presentes en estos jóvenes. Para comenzar, presentamos la siguiente tabla que refleja algunas de las actividades practicadas por jóvenes en el campo. 
Tabla 6

Actividades practicadas por la juventud rural

\begin{tabular}{|l|c|}
\hline ACTIVIDAD & PORCENTAJE \\
\hline Deportes (fútbol, ping pong, rodeo) & 59 \\
\hline Religiosas (grupos juveniles) & 21 \\
\hline Recreativas & 5 \\
\hline No hay actividades & 15 \\
\hline TOTAL & 100 \\
\hline
\end{tabular}

Fuente: Zapata, 2000:70.

Podemos ver entonces que en el campo se presenta una cultura juvenil bastante ligada a las actividades al aire libre, como es el deporte, en especial el fútbol y el rodeo, esta última un deporte relacionado con una cultura rural-campesina. A su vez un $20 \%$ manifiesta participar en grupos juveniles religiosos, lo cual refleja un fuerte capital social, al estar en su mayoría ligado estas actividades valores como la solidaridad, la unidad, la reciprocidad y la cooperación. En este sentido, existe un potencial de actividades a realizar, basado en las capacidades e interés de los jóvenes en participar, a pesar que muchas veces no cuentan con los medios para hacerlo. Incluso, los mismos jóvenes plantean opiniones e ideas interesantes que a ellos les gustaría realizar, como es el trabajo voluntario con niños y pre-jóvenes, y el establecimiento de redes con jóvenes de otras localidades, un potencial ciudadano que debe ser tomado en cuenta (INJUV, 1998).

Lo que haría falta aquí... juntar más a la juventud y darle más, otros objetivos... irnos a juntar con unos muchachos de Mulchén, hacer una convivencia, una cosa así...

Los mismos niños chicos... se empezaría con eso... en el verano no hacen nada, solamente están en la casa, entonces se harían otras actividades... le reparto dos pastillas a cada niño que venga y se juegue con ellos, cosas así, pero no se puede porque no hay unión en eso.

En cuanto a la participación política, presentamos a continuación los datos de registros electorales nacionales juveniles. Llama la atención de que a pesar de ser sólo un $40 \%$ los jóvenes rurales inscritos electoralmente en el país, es un porcentaje levemente mayor que la juventud urbana, expresando tal vez una mayor interés hacia la política, y en este sentido, una mayor conciencia ciudadana. 
Tabla 7

Jóvenes inscritos en los registros electorales (porcentaje)

\begin{tabular}{|c|c|c|}
\hline \multirow{2}{*}{$\begin{array}{c}\text { INSCRIPCIÓN } \\
\text { AL REGISTRO }\end{array}$} & URBANA & LOCALIZACIÓN \\
\cline { 2 - 3 } & 38.3 & 40.8 \\
\hline Sí & 61.7 & 59.2 \\
\hline No & 100.0 & 100.0 \\
\hline TOTAL & \multicolumn{2}{|c|}{} \\
\hline
\end{tabular}

Fuente: INJUV, 2002.

Un tema clave relacionado muy estrechamente con la ciudadanía es el de liderazgo, pues como veremos más adelante, puede ser una base importante para el fortalecimiento de procesos de participación grupal y comunitaria. En el contexto de la juventud rural, es un tema que todavía se encuentra impregnado por los modelos tradicionales de liderazgo imperantes en la sociedad chilena (autoritarios y clientelistas), los cuáles impiden el desarrollo de un nuevo modelo entre los dirigentes jóvenes. Tal es como lo plantea el resultado de grupos focales con dirigentes juveniles rurales, como vemos en las siguientes conclusiones. Sin embargo, la conciencia de estas limitaciones por parte de los mismos jóvenes rurales es ya un potencial clave para la generación de oportunidades para la reorientación de estas conductas sociales en pos del fortalecimiento de la democracia local: i) Hay una carencia de líderes, y líderes positivos, en los grupos juveniles y comunidades rurales. ii) Esta falta de un rol de liderazgo se debe al hecho que los jóvenes son «pasajeros» en el ámbito rural. iii) Los líderes juveniles deben aprender a hacer proyectos productivos, a ayudar a sus familias y comunidad. iv) Deben aprender a elegir a sus líderes, que sean «comprometidos». v) Deben superar los resabios de «autoritarismo» y control en los puestos de poder. vi) Los profesionales municipales y del área rural deben tener una actitud de «caminar con la gente», y no una lógica de mercado o de «oficina». Que los acompañen y les dediquen su tiempo (Zapata, 2000:107-109).

Podemos decir entonces que es urgente la definición de políticas sociales que permitan el surgimiento de un modelo alternativo de liderazgo, que potencie la ciudadanía juvenil rural. Además, tiene estrecha relación con la permanencia de la juventud en el campo, y la búsqueda de alternativas educacionales y laborales que se adapten a su estilo de vida.

Por último, otro tema que generalmente es relacionado con la realidad de la juventud rural es la pobreza, ya que en el país las cifras de pobreza general reflejan una tasa más alta en las zonas rurales. De 
todos modos, a pesar de ser mayor la pobreza en el mundo rural y entre la juventud rural en comparación con la urbana, medida de acuerdo con la línea de pobreza y las limitaciones que este instrumento plantea, en los últimos diez años ha habido una reducción considerable de la pobreza e indigencia (de un $47 \%$ a un $29 \%$, aproximadamente), como refleja la siguiente tabla.

Tabla 8

Población de 15 a 29 años, total nacional, por línea de pobreza, según zona, 1987- 1998

(porcentajes)

\begin{tabular}{|c|c|c|c|c|c|}
\hline AÑO & ZoNA & IndigENTE & $\begin{array}{c}\text { PoBRE } \\
\text { NO INDIGENTE }\end{array}$ & No POBRE & TOTAL \\
\hline \multirow{3}{*}{1987} & Urbana & 16,0 & 28,7 & 55,3 & 100 \\
\cline { 2 - 6 } & Rural & 16,3 & 31,0 & 52,7 & 100 \\
\cline { 2 - 6 } & Total & 16,1 & 29,2 & 54,8 & 100 \\
\hline \multirow{3}{*}{1996} & Urbana & 4,7 & 16,0 & 79,3 & 100 \\
\cline { 2 - 6 } & Rural & 8,9 & 19,6 & 71,4 & 100 \\
\cline { 2 - 6 } & Total & 5,4 & 16,6 & 78,0 & 100 \\
\hline \multirow{3}{*}{1998} & Urbana & 4,7 & 15,2 & 80 & 100 \\
\cline { 2 - 6 } & Rural & 8,3 & 16,9 & 74,9 & 100 \\
\cline { 2 - 6 } & Total & 5,2 & 15,4 & 79,3 & 100 \\
\hline
\end{tabular}

Fuente: MIDEPLAN, 1999 y 1998.

De todos modos, aún queda mucho por hacer en esta área, pues la pobreza juvenil rural, junto con la indigencia, abarcan a casi un $30 \%$ de la juventud del país, lo cual es casi un tercio de la población juvenil. Estos datos se corresponden con la situación de pobreza general rural en el país, donde existe aún casi un 30\% entre pobres e indigentes (CEPAL, 2002). De este modo, es todavía urgente diseñar políticas dirigidas a la superación de la pobreza juvenil rural, aunque el concepto de pobreza debe ser considerado en una forma más integral, al igual que las políticas mismas, ya que no es sólo el reflejo de una realidad económica. Tal como Max-Neef, Elizalde y Hopenhayn mencionan, es posible hablar de «pobrezas», de acuerdo con su propuesta de Desarrollo a Escala Humana, donde estipulan una serie de necesidades humanas fundamentales. ${ }^{2}$ A su vez, el economista ganador del premio

2 Estas necesidades son: subsistencia, protección, afecto, entendimiento, participación, ocio, creación, identidad y libertad, según la cultura y el momento histórico en que se encuentren. Max-Neef, Elizalde y Hopenhayn; 1986:27. 
Nobel, Sen (1999), considera a la pobreza como la «privación de capacidades», por lo que su superación depende de políticas integrales dirigidas también al desarrollo de capacidades y capital social.

\section{CAPITAL SOCIAL JUVENIL RURAL}

Después de analizar los potenciales y desafíos de la juventud rural en Chile, a continuación desarrollaremos algunos conceptos claves para comenzar a pensar en posibles programas que puedan basarse en éstos potenciales que presenta la juventud rural, para verlos como solución y no como problema. Luego citaremos dos casos de proyectos innovativos que nos pueden ayudarnos a visualizar estos conceptos en la acción.

Un concepto que nos permite operacionalizar las capacidades ciudadanas en el ámbito de los emprendimientos individuales y colectivos es el de capital social. En base a la tipología de Durston (2002:41-42), y una sistematización de la realidad expresada en las investigaciones anteriormente citadas (Durston y Duhart, 2003), podemos confeccionar la siguiente tabla.

Tabla 9

Capital social juvenil rural

\begin{tabular}{|c|c|c|}
\hline \multicolumn{2}{|l|}{ TIPO } & EJEMPLO \\
\hline \multicolumn{2}{|c|}{ Individual (diádico) } & $\begin{array}{l}\text { Amigos. Socios de emprendimientos diádicos. «Compadres». } \\
\text { Contactos personales con individuos de agencias externas y } \\
\text { patrones de trabajo temporal. Liderazgo. Vecindad. }\end{array}$ \\
\hline \multicolumn{2}{|c|}{$\begin{array}{l}\text { Grupal } \\
\text { (3 a } 10 \text { personas) }\end{array}$} & $\begin{array}{l}\text { Amigos/grupo de pares. La familia, nuclear y extendida. } \\
\text { Emprendimientos grupales y familiares. Grupos deportivos } \\
\text { (equipos de fútbol). Grupos de trabajo temporal. Recreación. }\end{array}$ \\
\hline \multicolumn{2}{|c|}{$\begin{array}{l}\text { Comunitario } \\
\text { (organizaciones } \\
\text { formales e informales) }\end{array}$} & $\begin{array}{l}\text { Organizaciones deportivas, religiosas y comunitarias. } \\
\text { La escuela y la comunidad escolar (alumnos, profesores, } \\
\text { administrativos). Liderazgos y dirigentes juveniles. Niños, } \\
\text { pre-jóvenes con quienes se relacionan. }\end{array}$ \\
\hline \multirow[t]{2}{*}{ Externo } & Puente & $\begin{array}{l}\text { Ligas deportivas (fútbol, rodeo, etc.). Federaciones de } \\
\text { organizaciones sociales y religiosas. Iglesias y sus redes } \\
\text { sociales. Emigración temporal y relaciones con otros } \\
\text { jóvenes rurales. Las ciudades donde se emigra y los con- } \\
\text { tactos establecidos. Jóvenes de localidades vecinas. }\end{array}$ \\
\hline & Escalera & $\begin{array}{l}\text { Relaciones con funcionarios y agencias estatales, munici- } \\
\text { pales, ONG's, fundaciones, y su apoyo para iniciativas } \\
\text { locales (FOSIS, INDAP, PRODESAL, IER, FUCOA, etc.). }\end{array}$ \\
\hline
\end{tabular}

Fuente: elaboración propia del autor. 
De acuerdo con esta tabla, existen muchos ejemplos de relaciones de reciprocidad, cooperación y confianza entre la juventud rural, así como con instituciones externas. Sin embargo, lo que es crucial es buscar formas de potenciar el capital social identificado, tanto a nivel individual, grupal y comunitario, como para desarrollar emprendimientos juveniles que impulsen el desarrollo en las comunidades rurales. Como vimos, existen ideas de proyectos comunitarios con niños y pre-jóvenes, así como entre los mismos jóvenes y una serie de actividades deportivas y recreativas, lo cual expresa capacidades de participación importantes. También hay un deseo que alguien los ayude y unifique en la generación de estas iniciativas, lo cual además se cruza con el diagnóstico negativo acerca del liderazgo entre los dirigentes juveniles. Habría una falta de líderes positivos, y que superen los modelos autoritarios, paternalistas y clientelistas, los cuales finalmente promueven el individualismo. En este sentido, ¿por qué no pueden ser los mismos jóvenes rurales quienes se levanten para llevar a cabo estos proyectos comunitarios en su entorno? ¿Acaso no poseen capacidades y pueden ser una solución para sus problemas? Para esto, fortalecer las formas de liderazgo comunitario y horizontal, como una distinción necesaria para ser actor social, que a su vez promueva la participación y el capital social, parece ser una línea de acción a seguir.

\section{LIDERAZGO}

Es necesario entonces presentar algunas reflexiones sobre el tema del liderazgo y nuevas propuestas para su desarrollo. Una definición muy interesante que nos permite evaluar los distintos modelos de liderazgo es la siguiente: «al hablar de liderazgo, siempre debemos hacerlo en el marco del funcionamiento de un grupo. El liderazgo no puede existir en un vacío. Existe en el contexto de un grupo» (Anello y Hernández, 1993:37-39). Un grupo tendría tres funciones principales: conservar y fortalecer la unidad del grupo; llevar a cabo las tareas para las cuales el grupo fue creado; desarrollar las potencialidades de los miembros del grupo.

Los diferentes modelos de liderazgo podrían ser evaluados según el grado en que contribuyen al logro de estas funciones de un grupo. De este modo, si algún modelo de liderazgo obstaculiza alguna de éstas, no sería un estilo deseable. 
Tabla 10

Modelos tradicionales de liderazgo

\begin{tabular}{|c|c|c|c|}
\hline \begin{tabular}{|c|} 
MODELOS \\
PREVALECIENTES \\
DE LIDERAZGO
\end{tabular} & $\begin{array}{c}\text { CONDUCTA QUE } \\
\text { CARACTERIZA EL } \\
\text { MODELO MENTAL DE } \\
\text { LIDERAZGO } \\
\end{array}$ & $\begin{array}{c}\text { REACCIÓN DE LOS } \\
\text { MIEMBROS DEL } \\
\text { GRUPO AL } \\
\text { LIDERAZGO } \\
\end{array}$ & $\begin{array}{c}\text { EFECTO EN EL } \\
\text { FUNCIONAMIENTO } \\
\text { DEL GRUPO }\end{array}$ \\
\hline Autoritario & $\begin{array}{c}\text { Da órdenes, espera } \\
\text { obediencia inmedia- } \\
\text { ta, exacta y sin } \\
\text { cuestionamiento a } \\
\text { su autoridad. } \\
\end{array}$ & \begin{tabular}{|c|} 
Sienten rebeldía y \\
resentimiento hacia \\
el líder, expresado \\
de manera directa o \\
indirecta. \\
\end{tabular} & $\begin{array}{l}\text { El grupo no cumple su } \\
\text { función de mantenerse } \\
\text { unidos, y tampoco se } \\
\text { desarrolla el potencial } \\
\text { de sus miembros. }\end{array}$ \\
\hline Paternalista & $\begin{array}{c}\text { Tiene sincera pre- } \\
\text { ocupación hacia los } \\
\text { miembros del grupo, } \\
\text { pero él resuelve los } \\
\text { problemas como un } \\
\text { padre sobreprotec- } \\
\text { tor. }\end{array}$ & $\begin{array}{c}\text { Se acostumbran a } \\
\text { este tipo de lideraz- } \\
\text { go, ya que es muy } \\
\text { cómodo. Se resisten } \\
\text { a los cambios que } \\
\text { lleven a que ellos } \\
\text { asumen las respon- } \\
\text { sabilidades. } \\
\end{array}$ & $\begin{array}{c}\text { Básicamente los miem- } \\
\text { bros no desarrollan sus } \\
\text { potenciales, ya que se } \\
\text { produce una dependen- } \\
\text { cia extrema hacia el } \\
\text { líder. Después, es muy } \\
\text { difícil hacer que asuman } \\
\text { un rol más protagónico. }\end{array}$ \\
\hline Sabelotodo & $\begin{array}{l}\text { La persona de ma- } \\
\text { yor conocimiento se } \\
\text { jacta de sus conoci- } \\
\text { mientos y habilida- } \\
\text { des, con una actitud } \\
\text { de superioridad. } \\
\text { Ridiculiza a otros. }\end{array}$ & $\begin{array}{c}\text { Crea sentimientos } \\
\text { de inferioridad en } \\
\text { los otros miembros } \\
\text { del grupo. Miedo. }\end{array}$ & $\begin{array}{l}\text { Hay una baja de partici- } \\
\text { pación al interior del } \\
\text { grupo por temor a la } \\
\text { ridiculez, y a su vez la } \\
\text { unidad del grupo se } \\
\text { rompe por la falta de } \\
\text { integración. Los miem- } \\
\text { bros no se desarrollan. }\end{array}$ \\
\hline Manipulador & $\begin{array}{l}\text { Aparenta pensar en } \\
\text { el bienestar de los } \\
\text { demás, cuando } \\
\text { oculta sus intereses } \\
\text { personales. }\end{array}$ & $\begin{array}{l}\text { Engaña y manipula } \\
\text { a los miembros del } \\
\text { grupo, usando la } \\
\text { demagogia y otros } \\
\text { medios. }\end{array}$ & $\begin{array}{c}\text { Las personas pierden } \\
\text { confianza en el líder, no } \\
\text { se desarrollan y el grupo } \\
\text { no logra los objetivos } \\
\text { para los cuales fue } \\
\text { creado. Se daña el } \\
\text { sentido de cooperación } \\
\text { en el grupo. }\end{array}$ \\
\hline
\end{tabular}

Fuente: basado en Anello y Hernández, 1993:39-50.

Estos modelos tienen mucha semejanza con las críticas que los dirigentes rurales juveniles realizaban hacia los tipos del liderazgo existentes entre ellos. ¿Será que los jóvenes rurales están adoptando estos mismos modelos tradicionales de liderazgo? ¿Acaso no son estos modelos los que ellos observan entre los dirigentes sociales, líderes políticos y patrones de fundo en el «mundo» adulto rural? Es por es este motivo que es urgente la búsqueda y desarrollo entre la juventud rural de un modelo alternativo de liderazgo que promueva el desarrollo 
general de las áreas rurales, tradicionalmente dependientes de líderes externos, paternalistas y autoritarios.

Un modelo alternativo de liderazgo muy interesante es el modelo de liderazgo moral/transformacional, orientado al servicio y centrado en el desarrollo de capacidades. El modelo inicia en las personas un proceso de aprendizaje individual enfocado al desarrollo de capacidades a través de un esfuerzo sistemático en la transformación de modelos mentales estáticos en marcos conceptuales evolutivos, comprendiendo conceptos, adquiriendo nuevas actitudes, y desarrollando destrezas y cualidades. Las capacidades se dividen en tres tipos: para la transformación personal, para la transformación de relaciones interpersonales, y para la transformación social.

a) Capacidades para la transformación personal: i) Capacidad de evaluar fortalezas y debilidades: la autoevaluación. ii) Capacidad de aprender de la reflexión sistemática sobre la acción dentro de un marco conceptual consistente y evolutivo. iii) Capacidad de tomar iniciativa de manera creativa y disciplinada. iv) Capacidad de mantener el esfuerzo, perseverar y superar los obstáculos. v) Capacidad de superar las tendencias individualistas: la autodisciplina. vi) Capacidad de manejar los asuntos y responsabilidades con rectitud de conducta. vii) Capacidad de pensar sistémicamente en la búsqueda de soluciones.

b) Capacidades para la transformación de las relaciones interpersonales: i) Capacidad de imbuir con principios éticos los pensamientos y acciones propias. ii) Capacidad de alentar a otros y de trabajar en equipo. iii) Capacidad de participar en la consulta de toma de decisiones colectivas. iv) Capacidad de ser un miembro responsable del grupo familiar y la comunidad. v) Capacidad de promover y construir unidad en diversidad.

c) Capacidades que contribuyen a la transformación social: i) Capacidad de crear una visión de un futuro deseado basada en principios. ii) Capacidad de comprender las relaciones de dominación y de contribuir a su transformación en relaciones basadas en la reciprocidad, la cooperación y el servicio mutuo. iii) Capacidad de contribuir como actor social juvenil al establecimiento de la justicia. iv) Capacidad de comprometerse con el proceso de potenciar actividades educativas. v) Capacidad de servir en las instituciones de la sociedad de manera que ayude a sus miembros a desarrollar y usar sus talentos y capacidades en servicio a la comunidad.

Proyectos e iniciativas que incorporen estos conceptos, como los de capital social y un modelo alternativo de liderazgo, son necesarios 
para potenciar a la juventud rural, para que ésta se constituya en una solución para sus problemas, en alianza con otros actores de la sociedad (Estado, jóvenes urbanos, adultos, etc.). A continuación citaremos dos experiencias muy innovativas desarrolladas en América Latina.

\section{UNIVERSIDAD RURAL/SISTEMA DE APRENDIZAJE TUTORIAL}

El primer proyecto innovador que queremos analizar es el de la Universidad Rural, creada en 1974 por un grupo de científicos y profesionales de la Fundación para la Aplicación y Enseñanza de las Ciencias (FUNDAEC) en región del Cauca del norte de Colombia. Desde entonces, este proyecto ha evolucionado y crecido enormemente, siendo reconocido por el Ministerio de Educación, e incluso difundiendo el modelo a otros países y continentes (Ecuador, Brasil, Guatemala, México, Honduras y Zambia), obteniendo reconocimiento mundial al ser premiado por el Club de Budapest, junto con otras tres iniciativas mundiales, como un proyecto educativo radicalmente novedoso.

En la base del modelo o marco conceptual de la Universidad Rural se encuentra la afirmación que la capacidad de un pueblo de participar en la generación y aplicación del conocimiento es un componente esencial de su proceso de desarrollo. En este proceso tendría un lugar central la ciencia, pero tanto el conocimiento universal como el propio o local, como expresión de la dimensión técnica de la cultura. En realidad, la interacción entre ambos sería lo que generaría un proceso dinámico de aprendizaje y desarrollo. En este sentido, la Universidad Rural es un espacio donde interactúan en forma saludable dos sistemas de conocimiento: uno moderno y uno tradicional, perteneciente a la gente de la región.

Y este concepto de la universidad como «espacio» no es un mero símbolo. De hecho, el concepto de Universidad Rural va más allá de un «edificio», ya que como hemos visto también en el caso de Chile, el determinar la educación en la existencia de edificio donde realizarlo es muy limitante para las zonas rurales, donde se encuentran muchos lugares distantes o aislados, o a su vez no existen los recursos económicos, tanto locales o estatales, para construirlos. Entonces, la Universidad Rural utiliza un sistema de aprendizaje flexible y que se adapta al mundo rural, así como al ciclo de vida rural, el cual es un Sistema de Aprendizaje Tutorial (SAT).

El SAT es un sistema de educación a distancia basado en la presencia de un tutor capacitado que vive en o cerca de las comunidades 
o aldeas rurales donde hay jóvenes rurales, y les ayuda a avanzar por un juego de 28 textos de estudio, a través de encuentros de 10 horas a la semana, hasta completar todo el programa. Este proceso puede durar entre tres a cuatro años, según el ritmo de vida de cada grupo, permitiendo a los jóvenes trabajar en el predio familiar e incluso realizar trabajos temporales. El horario es flexible, siendo establecido por cada grupo y su tutor, y puede ser en las tardes, noches o fines de semana. Lo importante es reunirse y avanzar en el aprendizaje, donde también se realizan actividades de aplicación práctica del conocimiento (Arbab, Correa y Valcárcel; 1988:14-17). Los principios pedagógicos del SAT son los siguientes: i) Fortalecimiento de la relación escuela-comunidad. ii) La integración (contenidos, estudio-trabajo, teoríapráctica, proyectos productivos). iii) Pertenencia, el arraigo y el compromiso de los agentes educativos con sus comunidades. iv) Flexibilidad. v) Capacitación, seguimiento y calificación permanentes. vi) Formación para la laboriosidad y el servicio. vii) Contenidos académicos adecuados a las necesidades del campo (Villegas, 2000).

Los contenidos del SAT equivalen a los años de enseñanza media, y posteriormente entrega posibles carreras universitarias y licenciaturas a seguir. Los textos, además de cubrir las áreas de conocimiento enseñadas tradicionalmente en los sistemas de educación, orientan a los estudiantes en una serie de actividades en la comunidad, relacionadas con la salud, la agricultura, la producción animal, la educación y la organización, centrando las actividades de aplicación y experimentación en el entorno comunitario así como en la búsqueda de sistemas alternativos de producción en pequeña parcela. Es un currículum educacional en armonía con los intereses y la realidad de la juventud rural, permitiéndoles conectar los conocimientos aprendidos (biología, matemáticas, historia, química, física, etc.) a su entorno. Tiene un énfasis importante en las actividades dirigidas al servicio y desarrollo local, graduándose los estudiantes a lo largo del programa en Impulsor del Bienestar Rural, Técnico en Bienestar Rural y Bachillerato en Bienestar Rural (Arbab, Correa y Valcárcel; 1988).

A su vez, como decíamos, puede conducir a un programa de educación universitaria, ya sea Licenciado en Educación Rural o Ingeniería para el Bienestar Rural. En este sistema, los alumnos son evaluados por un evaluador externo, ya que el tutor se compromete mucho con el proceso de aprendizaje. De acuerdo con Arbab, uno de los creadores de la Universidad Rural: 
No hay duda alguna que en este momento el SAT desarrolla en sus estudiantes capacidades que sobrepasan el nivel intelectual que le ha asignado a la juventud campesina una sociedad llena de prejuicios, y unas destrezas útiles para desempeñar una labor efectiva en la parcela familiar y prestar un servicio de valor a la comunidad (Arbab, Correa y Valcárcel; 1988:16).

El SAT comenzó primero como un proyecto más localizado de FUNDAEC, pero al extenderse por el país adoptó un sistema de contratación tripartita, cofinanciada por los departamentos estatales regionales en conjunto con los municipios, siendo ejecutado por organismos no gubernamentales. El número de alumnos por grupo oscila entre 15 a 25, teniendo menos gastos que un sistema tradicional de educación, lo cual ha facilitado su expansión. Una de las mejores formas de evaluar este sistema de educación es a través de sus logros, los cuáles enumeramos a continuación: i) Generación de un compromiso con el desarrollo de la región entre los habitantes rurales. ii) El desarrollo de sistema apropiados de producción y de tecnologías, así como de fortalecimiento organizacional. iii) La consolidación de actores sociales, especialmente mujeres y jóvenes, que desarrollan actos de servicio a la comunidad. iv) La participación de la población en un proceso de aprendizaje y de búsqueda científica para el mejoramiento de su entorno. v) Fortalecimiento del autoestima de los jóvenes y el impulso de un desarrollo en armonía con su identidad. vi) Permite que los jóvenes puedan seguir trabajando en sus predios familiares o en las cosechas temporales, mientras avanzan en sus estudios: se adapta al ciclo de vida del joven rural. vii) En treinta años se ha expandido a 17 regiones o provincias del país, con 30.862 estudiantes. viii) Ampliación de la cobertura educativa en el sector rural mediante alianzas Estadosociedad civil. ix) Generación de empleos rurales. x) Impacto en el desarrollo local mediante la promoción de proyectos sociales, educativos, culturales, productivos y de infraestructura. xi) Introducción de la aplicación de la ciencia para la interpretación del entorno de recursos y el mejoramiento de la producción, la sociedad y la cultura de la ruralidad, con un enfoque integral. xii) La formación en algunos casos de un comité consultivo integrado por organizaciones locales y ONG'S, como espacio de concertación entre el Estado y las instituciones de la sociedad civil.

Después de describir brevemente los rasgos generales y logros de este proyecto muy innovador, queda evidente que engloba muchos de los conceptos en torno al desarrollo que presentamos, así como responde a los desafíos y potenciales de la juventud identificados. Por un 
lado, es un proyecto que tiene como eje central la adquisición y aplicación del conocimiento, tanto propio como universal, por parte de la juventud rural, fortaleciendo de este modo su capacidad para seleccionar los elementos externos que desean adoptar y adaptar a su cultura, permitiéndoles en este sentido dirigir un estilo de desarrollo en armonía con su cultura e identidad. Este proceso además fortalece su autoestima, ayudándoles a desenvolverse en nuestro mundo globalizado, al desarrollar sus capacidades y despertar en ellos una conciencia de su valor propio. Además de entregar un sistema de educación a distancia que se adapta al ciclo de vida rural y el trabajo en el predio, tiene un currículo integral que abarca otras temáticas, como la organización y el servicio a la comunidad, fortaleciendo el capital social comunitario, y de este modo, la ciudadanía rural. Es un proyecto revolucionario por su concepto de universidad rural como un «espacio de aprendizaje» y no un edificio, que definitivamente empodera a la juventud rural. Sería muy interesante ensayar formas de aplicarlo en Chile, a través del mismo sistema tripartito de financiamiento y aplicación, a través de la acción conjunta entre las agencias estatales (FOSIS, INDAP, Mineduc), el municipio y los organismos no gubernamentales. Es un currículo fácil de aplicar y que ya ha sido adaptado a la realidad de varios países latinoamericanos.

\section{LIDERAZGO JUVENIL PARA EL SERVICIO AL BIEN COMÚN}

Otro proyecto innovador para juventud rural que deseamos proponer para la realidad chilena es el «Proyecto de liderazgo juvenil al servicio del bien común», de la Universidad Nur de Santa Cruz de la Sierra, Bolivia. Este proyecto está basado en el modelo de liderazgo moral/transformacional creado para el curso Capacitación de Agentes de Desarrollo Comunitario de su Instituto Superior de Educación Rural (ISER), al cual nos referimos cuando definimos un modelo alternativo de liderazgo. Ha sido aplicado tanto en contextos urbanos y rurales, en Bolivia como otros países latinoamericanos, y con el financiamiento de municipios y agencias internacionales, como el caso de UNICEF para la ciudad de Santa Cruz. En febrero de 2002 el programa de Liderazgo Juvenil de la Universidad Nur fue galardonado por el Banco Interamericano de Desarrollo (BID) como uno de las cuatro mejores «Prácticas Juveniles de Latinoamérica y el Caribe» en el área de la capacitación y voluntariado. 
A diferencia del SAT de FUNDAEC, es un proyecto con menos envergadura y más centrado en la formación de actores sociales o ciudadanía. Incluso, el SAT incorpora un módulo de liderazgo muy similar a éste, por lo que podría ser considerado como un componente del modelo de FUNDAEC. De todos modos, es un proyecto que puede sostenerse por sí mismo, como lo ha demostrado en diversos casos, y constituye un excelente ejemplo de proyecto inicial para ensayar políticas dirigidas a fortalecer el capital social y la ciudadanía juvenil rural, antes de generar un proyecto más integral y masivo como el caso del SAT.

Este proyecto tiene una estructura muy bien diseñada que busca desarrollar una capacitación transformadora en los jóvenes, ayudando a que desarrollen capacidades dirigidas al desarrollo comunitario y al fortalecimiento del capital social. Su objetivo general es crear una alternativa positiva para los jóvenes, generando oportunidades para desarrollar capacidades de liderazgo y para participar como voluntarios en equipos de servicio, en los cuales aprenderán como diseñar, implementar y evaluar proyectos de servicio comunitario, generando ciudadanía rural activa. En este sentido, recuerda mucho las reflexiones de Touraine en cuanto a la formación de un cuerpo de educadores de terreno y tutores que faciliten el surgimiento de actores sociales entre la juventud chilena (Touraine, 1996).

Presenta una estructura que no sólo se centra en la formación de actores sociales y ciudadanía para el futuro, sino que también en el presente, satisfaciendo las necesidades y anhelos de los jóvenes rurales como «jóvenes». Por este motivo, entre sus componentes principales tienen un lugar muy importante el uso de las artes y las actividades de socialización/entretención, basándose en los talentos e intereses de los jóvenes rurales que destacamos en nuestro diagnóstico de potenciales y desafíos. Además, contiene espacios dirigidos al fortalecimiento de las relaciones intergeneracionales (superando tensiones del ciclo de vida rural), para el acercamiento entre la comunidad y la escuela rural (capital social externo), y para la formación de redes con jóvenes de otros sectores o localidades (capital social de puente). De este modo, los componentes principales del proyecto son:

a) Talleres de capacitación en liderazgo juvenil: el concepto básico del liderazgo juvenil enfocado en la transformación es que todo adolescente y joven tiene la capacidad de ejercer una actitud proactiva para transformar tanto su propia vida como la de su comunidad.

b) Talleres de arte: el arte tiene el poder de motivar respuestas, motivar el cambio y promover la transformación personal y colectiva. 
Es un medio que ayuda a los jóvenes a definir y buscar soluciones a sus propios problemas, así como fortalecer su identidad y cultura locales.

c) Encuentros de integración intergeneracional: actividades paralelas que se realizarán para involucrar a otros actores, como son los profesores y los padres de familia, así como actividades de integración entre los jóvenes de las diferentes zonas involucradas. Otro tipo de encuentros son los festivales del deporte, festivales artísticos, y encuentros para el intercambio de experiencias entre los proyectos de servicio.

d) Proyectos de servicio: el joven que sirve contribuye a su propio proceso de transformación personal, así como a la transformación de su comunidad. Se considera que un líder es aquél que sirve más a su comunidad, sin esperar algo a cambio. Los proyectos de servicio prestan especial atención a los niños y pre-jóvenes, así como a la creación de un compromiso con el desarrollo de su región (Duhart, Masrour y Vieira; 2003).

$\mathrm{Al}$ ser un proyecto que persigue una capacitación transformadora entre los jóvenes, la metodología de aprendizaje utilizada involucra amplia participación, reflexión, consulta y acción. Los jóvenes aprenden a través de un proceso de reflexión sistemática acerca de sus acciones, donde el tutor tiene como rol ser facilitador del proceso de aprendizaje. La relación es de un diálogo de saberes, de un animador durante las sesiones. Los mismos proyectos de servicio comunitario abarcan desde un nivel simple hasta alcanzar mayores grados de complejidad, para lo cual también se capacita para el diagnóstico de necesidades basado en metodologías participativas. Son los jóvenes, analizando su propia situación, los que ofrecen la solución según las capacidades que van desarrollando (Duhart, Masrour y Vieira; 2003).

¿Pero cuál es realmente el trasfondo de una capacitación transformadora? El siguiente listado resume su propósito: i) Busca el desarrollo de capacidades, las cuales son una integración de conceptos, destrezas, actitudes y cualidades. ii) Persigue la transformación de modelos mentales no analizados en marcos conceptuales conscientes, consistentes y evolutivos. iii) Logra una motivación hacia la transformación fundamentada en un compromiso con principios e ideales. iv) Actúa en las tres dimensiones del ser humano necesarias para la transformación: la mente, la voluntad y la acción (Anello y Hernández, 1993).

Este proyecto tiene muchos potenciales para su aplicación en Chile y se justifica sobre algunos de los desafíos y potenciales identificados en nuestro diagnóstico sobre la juventud rural chilena. Por un lado, hay una demanda entre algunos de los dirigentes de un modelo 
de liderazgo menos autoritario y más comprometido con las comunidades locales, y este proyecto que aplica el paradigma del liderazgo moral/transformacional para jóvenes podría ser una buena forma de suplir esa demanda. Además, hay entre los jóvenes la demanda de más espacios de entretención y esparcimiento, y los festivales artísticos, encuentros deportivos y encuentros intergeneracionales podrían una alternativa de este tipo de actividades. Por último, entre algunos jóvenes hay ideas de proyectos comunitarios con niños y pre-jóvenes, así como de construcción de redes con jóvenes de otras localidades, y las actividades de servicio comunitario y de intercambio de experiencias del proyecto podrían ser una interesante forma de canalizar estas capacidades ciudadanas y de fortalecer el capital social individual, grupal y comunitario, contribuyendo fuertemente a la construcción de ciudadanía rural, en el marco de la nueva ruralidad y los procesos de descentralización.

\section{COMENTARIOS FINALES}

De este modo hemos presentado en este artículo un nuevo enfoque para aproximarse a la juventud rural. Esto es visualizarlos como solución y no como un problema, como suele ocurrir en los proyectos de desarrollo privados y estatales, donde por muy buenas intenciones que se tenga, el no centrarse en sus potenciales les impide tomar un rol protagónico en la definición de las opciones y la búsqueda de soluciones en sus vidas, y en este sentido, en la creación de una camino de desarrollo acorde con sus aspiraciones. Si creemos en la participación y el empoderamiento, es tiempo ya que pasemos del plano discursivo al de las acciones, y que busquemos soluciones integrales para problemas integrales, como son la pobreza y otros desafíos que debe enfrentar la juventud rural. La participación juvenil rural, basada en capacidades ciudadanas de liderazgo y servicio comunitario, puede ser un interesante aporte en este ámbito.

A modo de conclusión, podemos graficar las propuestas que deseamos compartir en el siguiente esquema que resume la secuencia, marco conceptual y lógica de ideas que las sustenta. Este esquema simboliza, con una pirámide invertida, el proceso de potenciación que los proyectos mencionados, u otros similares, pueden crear en la juventud rural, abordándolos como una solución más que como problemas en sí mismos, y alimentando los procesos de descentralización y desarrollo regional desarrollándose en forma simultánea. 


\section{Figura 1}

Interrelación, conceptos y propuestas

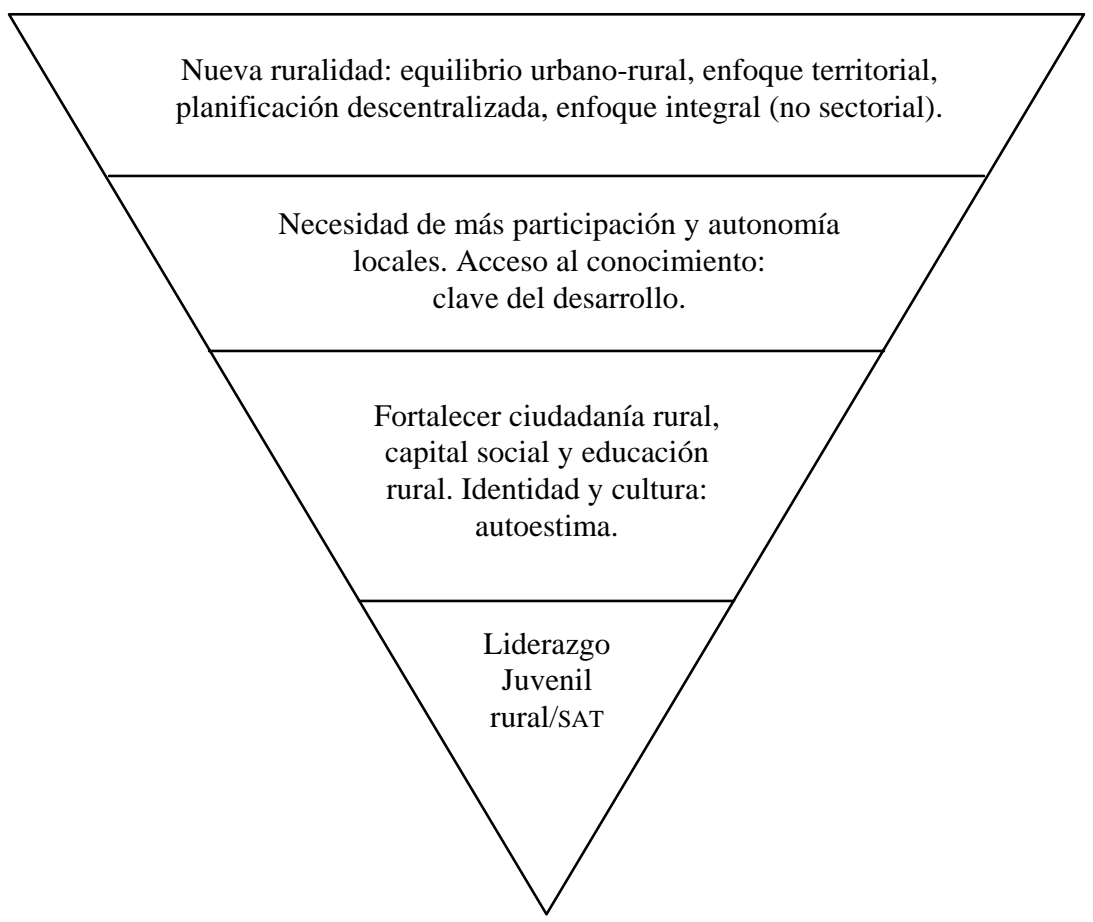

Cambios en los modelos mentales y en las metodologías que utilizamos para diseñar, aplicar y evaluar los programas dirigidos a la juventud rural, abriéndolos a su participación y a la generación de más «libertades» en sus vidas, parafraseando a Sen, asoman como senderos más adecuados a su realidad. Además, en la búsqueda de una mayor armonía con el mundo rural, una real sustentabilidad ambiental y un equilibrio urbano-rural en nuestra sociedad extremadamente urbanizada, los jóvenes rurales, y su gran diversidad (indígenas, diversidad regional, etc.), asoman como actores claves que pueden ayudarnos a dirigir mejor este proceso. 


\section{REFERENCIAS BIBLIOGRÁFICAS}

ANELLO, ElOY y JUANITA HeRNÁNDEZ (1993): «Liderazgo moral». Santa Cruz.

ARBAB, FARZAM (1991): La senda del aprendizaje en Latinoamérica: opción moral. Cali: Editorial Nur.

—, Gustavo CORREA y Francia de VALCÁRCEL (1988): «FUNDAEC: sus fundamentos y líneas de acción». CELATER y FUNDAEC.

CEPAL (2002): Panorama social de América Latina 2001-2002. Santiago: CEPAL.

— (1996): Juventud rural, modernidad y democracia en América Latina. Santiago: CEPAL.

DE LA MAZA, GonZALO (1999): «Los jóvenes temporeros de la VI Región. Un abordaje cualitativo». En: Jóvenes, cultura juvenil y subjetividad en el Chile de los 90. Estudios del INJUV Vol. 1. Santiago: INJUV.

DIRVEN, MARTINE (2002): Las prácticas de herencia de tierras agrícolas: ¿una razón más para el éxodo de la juventud?. Santiago: CEPAL.

DuHART, DANIEL; TIAGO MASROUR y VANDA VIEIRA (2003): «Construyendo ciudadanía rural: proyecto de liderazgo juvenil rural». Consultora Excelencia en Acción.

DURSTON, JOHN (2002): El capital social campesino en la gestión del desarrollo rural. Díadas, equipos, puentes y escaleras. Santiago: CEPAL.

— (1998): «La juventud y desarrollo rural: marco conceptual y contextual». Serie Políticas Sociales N²8. Santiago: CEPAL.

— y DANIEL DUHART (2003): «Formación y pérdida de capital social comunitario mapuche. Cultura, clientelismo y empoderamiento en dos comunidades, 1999-2002». Serie Políticas Sociales $\mathrm{N}^{\circ}$ 63. Santiago: CEPAL.

ECHEVERRI, RAFAEL y MARÍA PILAR RIBERO (2002): Nueva ruralidad. Visión del territorio en América Latina y el Caribe. San José: IICA.

INE (2003): Censo 2002. Resultados. Volumen I. Santiago: INE.

INJUV (2002): «Participación social y política de los y las jóvenes. Tercera encuesta nacional de juventud». Santiago: INJUV.

(1998): «Informe final: juventud rural. Regiones del Libertador, del Maule y del Bío-Bío». Santiago: INJUV. 
MAX-NEEF, MANFRED; ANTONIO ELIZALDE y MARTíN HOPENHAYN (1986): Desarrollo a escala humana. Estocolmo: CEPAUR, Fundación Dag Hammarskjöld.

MiDEPLAN (1999): «Resultados de la VII Encuesta de Caracterización Socioeconómica Nacional (CASEN 1998)». Santiago: MIDEPLAN.

- (1998): Serie de estadísticas sobre juventud. CASEN 1987 y 1996. Santiago: MIDEPLAN.

Sen, Amartya (1999): Freedom as Development. Oxford: Oxford University Press.

TOURAINE, AlAIN (1996): «Juventud y democracia en Chile». Revista Iberoamericana de Juventud $\mathrm{N}^{\circ} 1$. Madrid: OIJ.

VILLEGAS, LUZ (2000): «El sistema de aprendizaje tutorial (SAT). Una propuesta educativa para el desarrollo rural humano, armónico y sostenible». Conferencia del Banco Mundial «Educación y pobreza: incluyendo a los excluidos», 29-31 de mayo.

ZAPATA, SONIA (2000): Al encuentro del joven rural. Santiago: IICA. 